\title{
Sternoclavicular joint septic arthritis following paraspinal muscle abscess and septic lumbar spondylodiscitis with epidural abscess in a patient with diabetes: a case report
}

Nobuki Shioya*, Yoriko Ishibe, Shigenori Kan, Takayuki Masuda, Naoya Matsumoto, Gaku Takahashi, Hideyuki Makabe, Yasuhiko Yamada and Shigeatsu Endo

\begin{abstract}
Background: Septic arthritis of the sternoclavicular joint (SCJ) is extremely rare, and usually appears to result from hematogenous spread. Predisposing factors include immunocompromising diseases such as diabetes.

Case presentation: A 61-year-old man with poorly controlled diabetes mellitus presented to our emergency department with low back pain, high fever, and a painful mass over his left SCJ. He had received two epidural blocks over the past 2 weeks for severe back and leg pain secondary to lumbar disc herniation. He did not complain of weakness or sensory changes of his lower limbs, and his bladder and bowel function were normal. He had no history of shoulder injection, subclavian vein catheterization, intravenous drug abuse, or focal infection including tooth decay. CT showed an abscess of the left SCJ, with extension into the mediastinum and sternocleidomastoid muscle, and left paraspinal muscle swelling at the level of L2. MRI showed spondylodiscitis of L3-L4 with a contiguous extradural abscess. Staphylococcus aureus was isolated from cultures of aspirated pus from his SCJ, and from his urine and blood. The SCJ abscess was incised and drained, and appropriate intravenous antibiotic therapy was administered. Two weeks after admission, the purulent discharge from the left SCJ had completely stopped, and the wound showed improvement. He was transferred to another ward for treatment of the ongoing back pain.
\end{abstract}

Conclusion: Diabetic patients with S. aureus bacteremia may be at risk of severe musculoskeletal infections via hematogenous spread.

Keywords: Sternoclavicular joint, Septic arthritis, Spondylitis, Epidural abscess, Epidural anesthesia, Staphylococcus aureus

\section{Background}

Septic arthritis of the sternoclavicular joint (SCJ) is an unusual infection [1-3] which is not well understood, but appears to result from either hematogenous or contiguous spread [4]. Spondylodiscitis usually results from hematogenous spread of infections of the skin and subcutaneous tissues [5]. Common predisposing factors include immunocompromising diseases such as diabetes

\footnotetext{
* Correspondence: fukubuku@xg7.so-net.ne.jp

Department of Critical Care and Emergency, Iwate Prefectural Advanced Critical Care and Emergency Center, Iwate Medical University, 19-1 Uchimaru, Morioka 020-8505, Japan
}

[6,7]. Staphylococcus aureus is the most common pathogen in septic spondylitis and septic arthritis, and the most common portal of entry is through the skin.

\section{Case presentation}

A 61-year-old Japanese man was transported to our critical care and emergency center by ambulance with fever, exacerbation of pain in his lower back and both legs, and a painful mass over his left SCJ.

Approximately 3 months previously, he had consulted an orthopedic surgeon because of low back and leg pain. $\mathrm{He}$ had been diagnosed with disc herniation at L4-L5,

\section{Biomed Central}


and had been hospitalized for bed-rest and treatment. While hospitalized, he had received several intravenous injections of sodium salicylate, but no peripheral intravenous catheter had been inserted. About 2 months after discharge, he had been referred to our outpatient anesthesiology department because of ongoing leg pain. Two weeks before presentation, he had received his first epidural block using $6 \mathrm{ml}$ of $0.8 \%$ mepivacaine hydrochloride at the L4-L5 level, injected via the paramedian approach. Two days before presentation, a second epidural block using $5 \mathrm{ml}$ of $0.8 \%$ mepivacaine hydrochloride had been administered at the same level. The skin had been disinfected with $5 \%$ chlorhexidine solution prior to needle insertion on both occasions. His medical history included hypertension and diabetes, for which he was taking antihypertensive and hypoglycemic agents, respectively. He had no history of trauma, shoulder injection, subclavian vein catheterization, or intravenous drug abuse. He did not have any focal dental infection or signs of tooth decay. He was unable to walk, due to increased leg pain. The day prior to admission, he experienced a fever of $39^{\circ} \mathrm{C}$.

On examination, he was in moderate respiratory distress and mildly diaphoretic, with a blood pressure of 97/ $51 \mathrm{mmHg}$, pulse rate of 95 beats/min, respiratory rate of 28 breaths/min, temperature of $39.5{ }^{\circ} \mathrm{C}$, and oxygen saturation of $80 \%$ on room air. His oxygen saturation improved to $92 \%$ with oxygen administration (2 1/min by nasal cannula). Examination of the oral cavity and pharynx was normal, and there was no cervical lymphadenopathy. Chest examination was unremarkable except for swelling and severe tenderness over the left SCJ. Lumbar spine examination showed stiffness, with tenderness over the vertebrae. Movement of the lower back and pressure over the lumbar spine caused pain. The straight leg raising test and femoral nerve stretch test were inconclusive bilaterally because of lower back muscle spasm. His lower limb muscle power, knee and ankle reflexes, and sensation were normal. Bladder and bowel function were normal.

Laboratory testing showed the following results: plasma white blood cell count (WBC) $18,490 / \mathrm{mm}^{3}$, platelet count $541,000 / \mathrm{mm}^{3}$, hemoglobin $9.0 \mathrm{~g} / \mathrm{dl}, \mathrm{C}$ reactive protein $22.9 \mathrm{mg} / \mathrm{dl}$, fasting blood glucose $335 \mathrm{mg} / \mathrm{dl}$, glycosylated hemoglobin $8.1 \%$, blood urea nitrogen $23.7 \mathrm{mg} / \mathrm{dl}$, creatinine $0.73 \mathrm{mg} / \mathrm{dl}$, glutamicoxaloacetic transaminase $59 \mathrm{IU} / \mathrm{l}$, glutamic-pyruvic transaminase $62 \mathrm{IU} / \mathrm{l}$, cholinesterase 134 IU/l, alkaline phosphatase $600 \mathrm{IU} / \mathrm{l}$, lactate dehydrogenase $381 \mathrm{IU} / \mathrm{l}$, and creatine kinase 566 IU/l. US examination of the left SCJ suggested pyogenic arthritis with involvement of the sternocleidomastoid muscle. The chest X-ray was normal, and there were no abnormalities on ECG or cardiac US. CT showed erosion and abscess formation of the SCJ with extension of the abscess into the mediastinum (Figures $1 \mathrm{~A}$ and $1 \mathrm{~B}$ ) and sternocleidomastoid muscle (Figure $1 \mathrm{C}$ ). Abdominal CT showed swelling of the left paraspinal muscle at L1-L3 (Figure 2A). MRI showed spondylitis of the L3-L4 vertebrae (Figure 2B) with a focal epidural collection and L3-L4 discitis (Figure 2C).
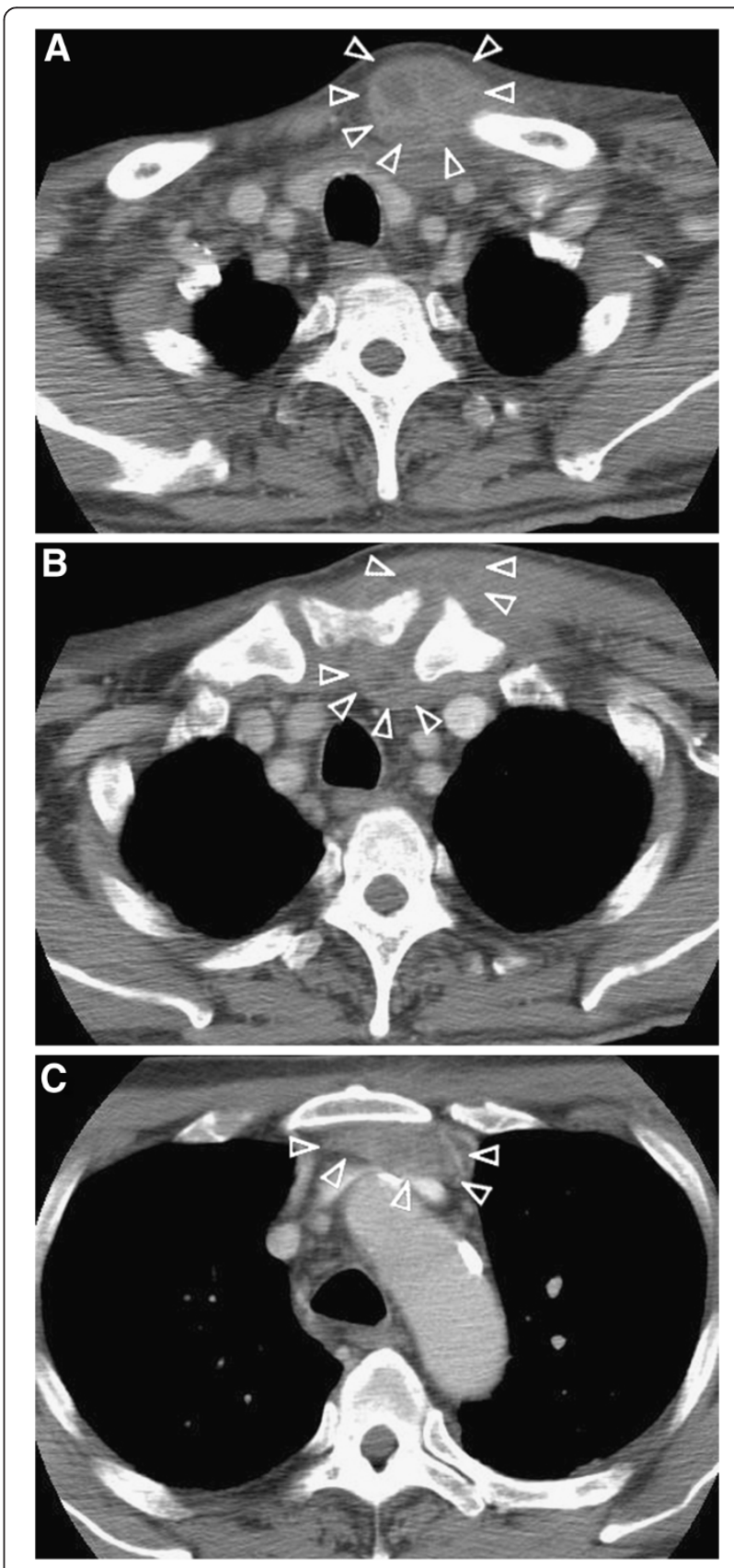

Figure 1 Thoracic CT scan: A CT scan using intravenous contrast shows an abscess (allow head: $\nabla$ ) around the left SCJ ( $A$ and $B$ ). The abscess is compartmental structure. The rim of the mass is slightly enhanced, but the center of the abscess is not enhanced. The abscess extends to the left sternocleidomastoid muscle $(\mathbf{A})$ and retrosternal region $(\mathbf{C})$. 

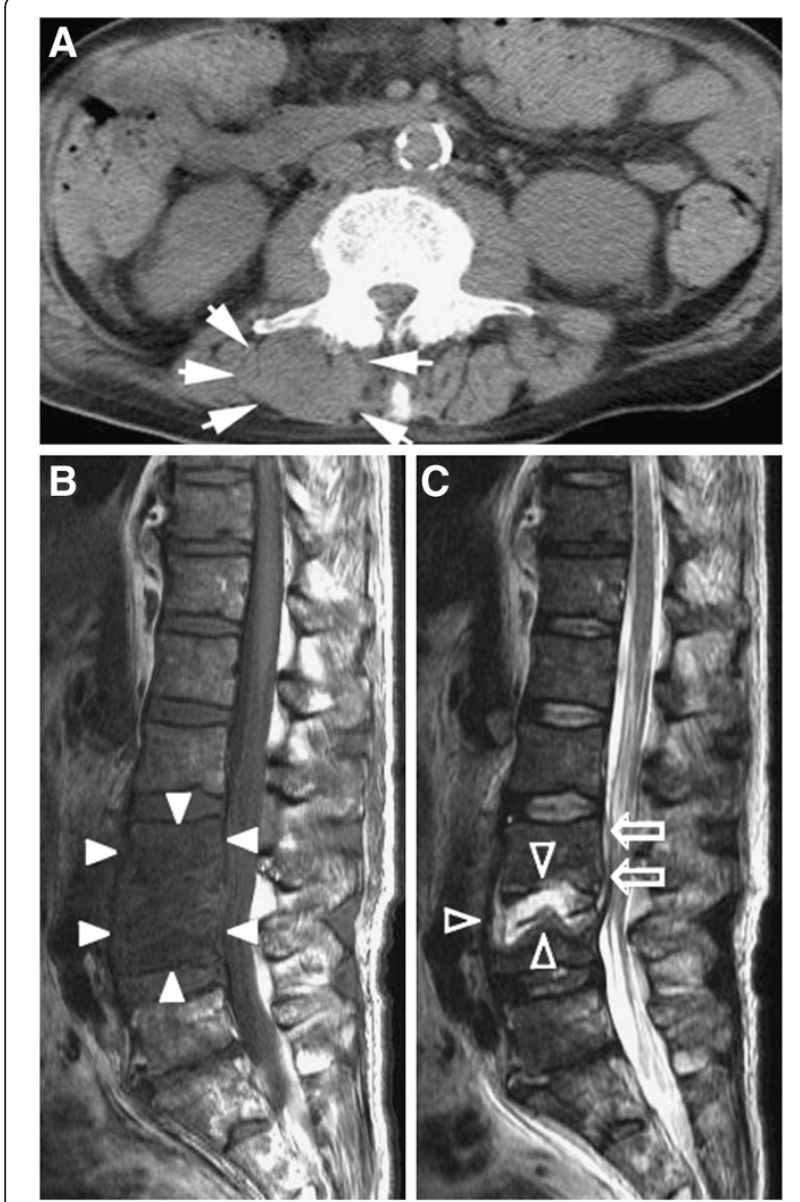

Figure 2 Lumbar CT scan and MRI: CT demonstrates swelling (white allow: $\uparrow$ ) of the left paraspinal muscle around the L2 level. (A) MRI reveals spondylitis (white allow head: $\mathbf{\Delta}$ ) lesions involving the $L 3-L 4$ vertebrae (B) with ventral contiguous epidural (framed allow: $\Uparrow$ ) and disc (framed allow head: $\nabla$ ) involvement (C).

Aspiration of the SCJ revealed a turbid fluid (approximately $3 \mathrm{ml}$ ) (Figure 3) with clusters of gram-positive cocci on microscopy. The SCJ was incised and drained, and the abscess cavity was enlarged to include the abscess of the left sternocleidomastoid muscle. Sulbactam/ ampicillin administration was started immediately. Aspirated pus, urine, and two sets of blood cultures all indicated S. aureus infection. According to the results of antibiotic susceptibility testing, the patient's antibiotic therapy was changed to cefotiam. After one week of antibiotic therapy, blood and wound cultures were negative for pathogens. Follow-up MRI clearly showed abscess formation in the left paraspinal muscle at L1-L3, but culture of fluid aspirated from the abscess showed no growth.

The patient's general condition improved significantly and his fever subsided after 4 days. The wound at the SCJ was irrigated daily. Twelve days after the onset of treatment, his plasma WBC was $7,050 / \mathrm{mm}^{3}$ with $78 \%$

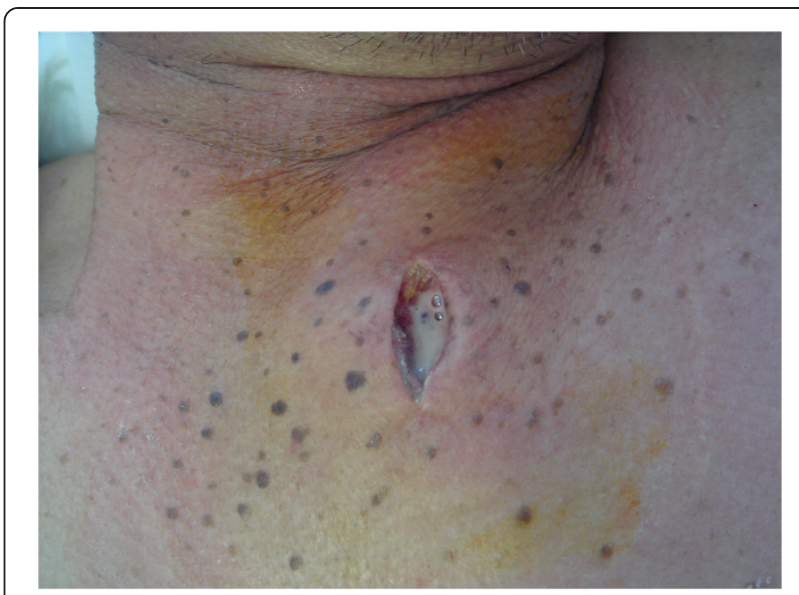

Figure 3 The left SCJ. Aspiration of the SCJ and culture of the obtained fluid yielded a growth of $\mathrm{S}$. aureus.

neutrophils. Two weeks after admission, there was a still a non-tender swelling over the left SCJ, but the purulent secretion had completely resolved. Neurological examination of the lower limbs was unchanged. Eight weeks after admission, he was transferred to the spinal surgery unit to undergo evaluation for operative treatment of his spondylitis and epidural abscess.

\section{Discussion}

It is likely that this patient's epidural block caused the paraspinal muscle abscess and nearby lumbar spondylodiscitis, and that this iatrogenic infection spread hematogenously to the SCJ.

Septic arthritis most commonly affects the weightbearing joints of the lower limb, which account for 61-79 $\%$ of all reported cases of septic arthritis [8]. The knee is the most commonly affected joint, followed by the hip, shoulder, wrist, ankle, and elbow. There is usually no limiting basement plate under the well-vascularized synovial membrane, facilitating the entry of hematogenously carried bacteria into the joint space [9].

Septic arthritis of the SCJ is extremely rare, comprising $0.5-1 \%$ of all joint infections [10], but results in abscess formation in $20 \%$ of cases $[11,12]$. The SCJ is the only joint connecting the trunk with the pectoral girdle, and is therefore involved in all major movements of the upper limb. The function of the articular disc on the clavicular side of the $\mathrm{SCJ}$ is to resist the compressive load [13]. SCJ infection can cause life-threatening complications, because the joint capsule is unable to distend and infection spreads beyond the joint quickly, leading to fistula formation, cutaneous abscess or, rarely, mediastinitis and superior vena cava syndrome $[14,15]$.

The pathogenesis of SCJ infection is not well understood, but it appears to result from either hematogenous or contiguous spread. Various factors have been 
identified as predisposing to the development of SCJ infections, including immunocompromising diseases such as diabetes, rheumatoid arthritis, renal dysfunction, and human immunodeficiency virus infection [6].The SCJ can be seeded with microorganisms via the subclavian vein following injection into the veins of the upper extremity or neck (including intravenous drug abuse), clavicular fractures, subclavian vein catheterization, or scratches or animal bites to the hand or arm $[6,16]$. SCJ infection is generally unilateral, affecting the right side in approximately $60 \%$ of cases. This difference in occurrence between sides is less apparent in intravenous drug abusers $[4,17]$. According to El Ibrahimi, bacteremia was the most commonly assumed mechanism of infection [17]. As in most joints, septic arthritis of the SCJ is most commonly caused by $S$. aureus, followed by pseudomonas species $[18,19]$. If an abscess develops, drainage and thorough debridement are necessary. Excision of the medial end of the clavicle, first rib, and manubrium may be required, which usually leaves a large chest wall defect, and exposes major vessels. This defect can be repaired with an advancement or rotational flap of the pectoralis major muscle [20].

Paraspinal muscle infection, a pyogenic infection of skeletal muscle, is rarely reported. Modes of infection include transcutaneous infection by needles or catheters, surgery, blunt trauma, and hematogenous spread from distant sites.

Spondylodiscitis, a term encompassing vertebral osteomyelitis, spondylitis, and discitis, is a rare medical emergency. Spinal epidural abscess is also uncommon, and requires early detection and appropriate treatment to prevent severe morbidity and mortality [21-24]. About 5\% of patients with spinal epidural abscesses die, usually because of uncontrolled sepsis, meningitis, or other underlying illnesses [22]. In $25-50 \%$ of cases, spondylodiscitis is associated with an epidural abscess or granulation tissue. Pyogenic spondylodiscitis with an epidural abscess may progress to a severe neurological deficit, especially if the diagnosis is established late and it is complicated by the development of an intramedullary abscess of the spinal cord [21].

Hematogenous osteomyelitis usually occurs in patients over 50 years of age and accounts for 3-5\% of all cases of osteomyelitis. The incidence of hematogenous osteomyelitis is estimated to be 4 to 24 per million per year in developed countries. Pathogens may infect the spine via three routes: hematogenous spread, direct external inoculation, and spread from contiguous tissues [25]. Spontaneous pyogenic spondylodiscitis usually spreads hematogenously from infections of the skin, subcutaneous tissues, or urinary tract [5]. The hematogenous arterial route predominates, allowing seeding of infection from distant sites to the vertebral column [25]. The most common causative pathogen is Staphylococcus aureus, followed in frequency by Brucella, Salmonella, and Mycobacterium tuberculosis [21,26]. The most likely portal of entry in cases of $S$. aureus infection is the skin (particularly from the skin creases between the toes). Unlike urinary tract infections caused by other pathogens, those caused by $S$. aureus are most often due to hematogenous dissemination. The presence of $S$. aureus in the urine, as in this case, therefore suggests hematogenous spread of infection [27]. S. aureus, Streptococcus species, and N. gonorrhoeae have a high degree of selectivity for the synovium, probably related to adherence characteristics and toxin production [9].

In adults, the vertebral intraosseous metaphyseal artery is an end-artery, and a septic embolism in a metaphyseal artery causes a large wedge-shaped infarct of a subdiscal area of bone. The subsequent spread of infection to the neighboring disc and vertebra creates the characteristic lesion of spondylodiscitis [25]. Ventrally located epidural abscesses in cases such as ours are usually associated with spondylitis and/or discitis [28]. Inoculation is most commonly iatrogenic following spinal surgery, lumbar puncture, or epidural procedures, accounting for $25-30 \%$ of cases in some spondylodiscitis series [25]. Mylona et al. described other sources of infection including the genitourinary tract (17\%), skin and soft tissue (11\%), intravascular devices $(5 \%)$, gastrointestinal tract $(5 \%)$, respiratory tract (2\%), and the oral cavity (2\%) [29]. They found that $12 \%$ of patients with pyogenic vertebral osteomyelitis also had infective endocarditis.

\section{Conclusion}

We present a patient with poorly controlled diabetes who developed S. aureus septic arthritis of the SCJ following spondylodiscitis. The most likely portal of entry in cases of $S$. aureus septic arthritis is the skin. We identified a recent epidural block as a potential iatrogenic source of infection in our case. It is noteworthy that diabetic patients with $S$. aureus bacteremia may be at risk of severe extended musculoskeletal infections.

\section{Consent}

Written informed consent was obtained from the patient for publication of this case report and any accompanying images. A copy of the written consent is available for review by the Editor-in-Chief of this journal.

\section{Competing interests}

The authors declare that they have no competing interests.

\section{Authors' contributions}

NS treated the patient and wrote the case report. YI, SK, TM, NM, GT, HM, and $Y Y$ were involved in the treatment of the patient. SE supervised the writing of this paper and made some major changes after reviewing the first versions. All authors read and approved the final manuscript. 


\section{Acknowledgments}

We would like to thank Prof. Endo who provided helpful comments and suggestions.

Received: 30 August 2011 Accepted: 24 May 2012

Published: 15 June 2012

\section{References}

1. Mikroulis DA, Verettas DA, Xarchas KC, Lawal LA, Kazakos KJ, Bougioukas GJ: Sternoclavicular joint arthritis and mediastinitis. A case report and review of literature. Arch Orthop Trauma Surg 2008, 128:185-187.

2. Mohyuddin A: Sternoclavicular joint septic arthritis manifesting as a neck abscess: a case report. Ear Nose Throat J 2003, 82:618-621.

3. Ho G Jr: Bacterial arthritis. Curr Opin Rheumatol 1991, 3:603-609.

4. Gillis S, Friedman B, Caraco Y, Blankstein A, Yellin A, Friedman G: Septic arthritis of the sternoclavicular joint in healthy adults. J Inter Med 1990, 228:275-278.

5. Kapsalaki E, Gatselis N, Stefos A: Spontaneous spondylodiscitis: presentation, risk factors, diagnosis, management, and outcome. Int J Infect Dis 2009, 13:564-569.

6. Chen WS, Wan YL, Lui CC, Lee TY, Wang KC: Extrapleural abscess secondary to infection of the sternoclavicular joint. J Bone Joint Surg Am 1993, 75:1835-1839.

7. Bajwa ZH, Ho C, Grush A, Kleefield J, Warfield CA: Discitis Associated with Pregnancy and Spinal Anesthesia. Anesth Analg 2002, 94:415-416.

8. Park AL, Dlabach JA: Infectious arthritis. In Campbell's Operative Orthopaedics. Edited by Canale ST. St. Louis: Mosby; 2003:685-711.

9. Shirtliff ME, Mader JT: Acute septic arthritis. Clin Microbiol Rev 2002, 15:527-544.

10. Ross JJ, Shamsuddin H: Sternoclavicular septic arthritis: review of 180 cases. Med Baltimore 2004, 83:139-148.

11. Chen WS, Wan YL, Liu CC, Lee TY, Wang KC: Extrapleural abscess secondary to infection of sternoclavicular joint. Report of two cases. $J$ Bone Joint Surg Am 1993, 75:1835-1839.

12. Wohlgetham JR, Newberg AH, Reed Jl: The risk of abscess from sternoclavicular septic arthritis. J Rheumatol 1988, 15:1302-1306.

13. Emura K, Arakawa T, Terashima T, Miki A: Macroscopic and histological observations on the human sternoclavicular joint disc. Anat Sci Int 2009, 84:182-188.

14. Asnis DS, Dhaliwal GS: Bilateral sternoclavicular joint septic arthritis presenting as cutaneous abscesses. Clin Infect Dis 1994, 19:964-966.

15. Koroscil TM, Valen PA: Sternoclavicular septic arthritis due to Haemophilus influenzae. South Med J 1990, 83:1469-1471.

16. Nitsche JF, Vaughan JH, Williams G, Curd JG: Septic sternoclavicular arthritis with Pasteurella multicoda and Streptococcus sanguis. Arhritis Rheum 1982, 25:467-469.

17. El Ibrahimi A, Daoudi A, Boujraf S, Elmrini A, Boutayeb F: Sternoclavicular septic arthritis in a previously healthy patient: a case report and review of the literature. Int J Infect Dis 2009, 13:e119-e121.

18. Lowy FD: Medical progress: staphylococcus aureus infections. N Engl J Med 1998, 339:520-532.

19. Ross JJ, Saltzman CL, Carling P, Shapiro DS: Pneumonococcal septic arthritis: review of 190 cases. Clin Infect Dis 2003, 36:319-327.

20. Pradhan C, Watson NF, Jagasia N, Chari R, Patterson JE: Bilateral sternoclavicular joint septic arthritis secondary to indwelling central venous catheter: a case report. J Med Case Rep 2008, 29(2):131.

21. Turgut M: Complete recovery of acute paraplegia due to pyogenic thoracic spondylodiscitis with an epidural abscess. Acta Neurochir (Wien) 2008, 150:381-386.

22. Darouiche RO: Spinal epidural abscess. N Engl J Med 2006, 355:2012-2020

23. Boström $A$, Oertel $M$, Ryang $Y$, Rohde $V$, Bürgel $U$, Krings $T$, Korinth $M$ : Treatment strategies and outcome in patients with non-tuberculous spinal epidural abscess-a review of 46 cases. Minim Invasive Neurosurg 2008, 51:36-42.

24. Reihsaus E, Waldbaur H, Seeling W: Spinal epidural abscess: a metaanalysis of 915 patients. Neurosurg Rev 2000, 23:175-204.

25. Gouliouris T, Aliyu SH, Brown NM: Spondylodiscitis: update on diagnosis and management. J Antimicrob Chemother 2010, 65(Suppl 3):11-24.

26. Priest DH, Peacock JE Jr: Hematogenous vertebral osteomyelitis due to Staphylococcus aureus in the adult: clinical features and therapeutic outcomes. South Med J 2005, 98:854-862.
27. Franklin DL: Staphylococcal Infections. In, Origins of Harrison's Principle of Internal Medicine. 17th editionEdited by Anthony SF. New York: McGraw-Hill Professional; 2008:872-879.

28. Oktenoglu T, Sasani M, Cetin B, Bozkus H, Ercelen O, Vural M, Ozer AF, Sarioglu AC: Spontaneous pyogenic spinal epidural abscess. Turk Neurosurg 2011, 21:74-82.

29. Mylona E, Samarkos M, Kakalou E, Fanourgiakis P, Skoutelis A: Pyogenic vertebral osteomyelitis: a systematic review of clinical characteristics. Semin Arthritis Rheum 2009, 39:10-17.

doi:10.1186/1471-227X-12-7

Cite this article as: Shioya et al: Sternoclavicular joint septic arthritis following paraspinal muscle abscess and septic lumbar spondylodiscitis with epidural abscess in a patient with diabetes: a case report. BMC Emergency Medicine 2012 12:7.

\section{Submit your next manuscript to BioMed Central and take full advantage of:}

- Convenient online submission

- Thorough peer review

- No space constraints or color figure charges

- Immediate publication on acceptance

- Inclusion in PubMed, CAS, Scopus and Google Scholar

- Research which is freely available for redistribution 